\title{
Association of carotenoids, tocopherols and vitamin $C$ in plasma with allergic rhinitis and allergic sensitisation in adults
}

\author{
Iris Kompauer ${ }^{1,2}$, Joachim Heinrich ${ }^{1, *}$, Guenther Wolfram ${ }^{3}$ and Jakob Linseisen ${ }^{4,5}$ \\ 'GSF - National Research Center for Environment and Health, Institute of Epidemiology, PO Box 1129, D-85758 \\ Neuherberg, Germany: ${ }^{2}$ Ludwig-Maximilians University of Munich, Institute of Medical Data Management, \\ Biometrics and Epidemiology, Munich, Germany: ${ }^{3}$ Department of Food and Nutrition, TU Munich, Munich, \\ Germany: ${ }^{4}$ Unit of Human Nutrition and Cancer Prevention, TU Munich, Munich, Germany: ${ }^{5}$ Division of Clinical \\ Epidemiology, German Cancer Research Centre, Heidelberg, Germany
}

Submitted 28 April 2005: Accepted 23 August 2005

\begin{abstract}
Objectives: Antioxidant nutrients like carotenoids, tocopherols and vitamin $\mathrm{C}$ have been suggested to protect against allergic rhinitis and allergic sensitisation but scientific evidence is scarce. The aims of the study were to measure the plasma concentration of six carotenoids, $\alpha$ - and $\gamma$-tocopherol and vitamin $\mathrm{C}$ as biomarkers of the intake, absorption and subsequent metabolism of these nutrients, and to assess their association with allergic rhinitis and sensitisation.

Method: Data from a cross-sectional study on representative dietary and lifestyle habits of the population of Bavaria, Germany, were analysed. The plasma levels of six carotenoids ( $\alpha$-carotene, $\beta$-carotene, lycopene, lutein/zeaxanthin, canthaxanthin and cryptoxanthin) as well as of $\alpha$-tocopherol, $\gamma$-tocopherol and vitamin $C$ were measured in 547 adults aged between 19 and 81 years. Participants with specific serum immunoglobulin $\mathrm{E} \geq 700 \mathrm{Ul}^{-1}$ were categorised as sensitised. The association of plasma antioxidant levels, allergic rhinitis and allergic sensitisation was assessed by means of unconditional logistic regression models.

Results: We observed a negative association between plasma total carotenoids and the prevalence of allergic rhinitis, with odds ratio (95\% confidence interval) of 1.13 $(0.54-2.39)$ for the second, $0.72(0.33-1.58)$ for the third and $0.44(0.19-1.03)$ for the fourth quartile of total carotenoids concentration $(P$ for trend $=0.0332$ ); results for lycopene failed to reach statistical significance $(P=0.0608)$. Other single carotenoids, tocopherols and vitamin $\mathrm{C}$ were unrelated to allergic rhinitis. Allergic sensitisation was negatively associated with plasma $\gamma$-tocopherol, with odds ratio $(95 \%$ confidence interval) of $0.92(0.51-1.65)$ for the second, $1.00(0.56-1.80)$ for the third and 0.45 $(0.23-0.88)$ for the fourth quartile of plasma $\gamma$-tocopherol concentration ( $P$ for trend $=0.0410)$. No other antioxidant was significantly related to allergic sensitisation.

Conclusions: High plasma carotenoid concentrations reflecting a diet high in various fruits and vegetables might have a protective effect on allergic rhinitis in adulthood.
\end{abstract}

\author{
Keywords \\ Antioxidants \\ Biomarkers \\ Allergic rhinitis \\ Allergic sensitisation
}

In the last few decades Western countries have witnessed increased prevalences of allergic diseases like hay fever and asthma ${ }^{1,2}$, although very recent results suggest a flattening of rising asthma incidence in affluent societies $^{3-5}$. Because dietary factors like the intake of antioxidants also changed during the same period, it has been postulated that antioxidants might influence the development of allergic diseases ${ }^{6-8}$. Additionally there is evidence that allergic diseases are mediated by oxidative stress, which results from an imbalance between reactive oxygen and nitrogen species and antioxidants?. These oxidants are capable of modifying lipids, proteins and nucleic acids ${ }^{10}$. All cells in the human body, including those of the respiratory tract, are constantly exposed to reactive oxygen and nitrogen species from endogenous and exogenous sources. Enzymatic as well as non-enzymatic antioxidants contribute to the antioxidant defence system in mammals. Carotenoids, vitamin E and vitamin $\mathrm{C}$ belong to the non-enzymatic antioxidants, and main food sources are fruit and vegetables as well as vegetable oils in the case of vitamin $\mathrm{E}$. Beside their antioxidant properties, vitamin $\mathrm{E}$ and vitamin $\mathrm{C}$ are supposed to have other antiallergic effects. Vitamin $\mathrm{E}$ is thought to act by inhibition of immunoglobulin E (IgE) production and membrane stabilisation ${ }^{11,12}$, vitamin $\mathrm{C}$ by inhibition of specific prostaglandin synthesis ${ }^{8,11}$. It is 
hypothesised that a low intake of these micronutrients might contribute to the rising prevalence rates of allergic diseases $^{7,9}$

Studies on the relationship between allergic sensitisation and respectively serum vitamin $\mathrm{E}^{13}$ and dietary intake of vitamin $\mathrm{E}^{14}$ show a negative association. The only study with prospective design on the influence of dietary intake of these micronutrients on hay fever reported a negative association of vitamin $\mathrm{E}$ intake and a positive association of $\beta$-carotene intake with adult onset of hay fever in ex- and current smokers, but no association of these antioxidants or vitamin C in non-smokers ${ }^{15}$.

In the present paper we focus on the contribution of carotenoids, tocopherols and vitamin $\mathrm{C}$ in plasma to allergic rhinitis and allergic sensitisation, and use the plasma concentration of these micronutrients as a marker of intake, absorption and subsequent metabolism.

\section{Materials and methods}

\section{Study design}

The Bavarian Nutrition Survey II (BVS II) was conducted between September 2002 and June 2003 to collect current and representative data on dietary and lifestyle habits of the Bavarian population. From the basic population of Bavaria (in the south of Germany) aged 13-81 years, 1050 persons were recruited by a three-stage random route sampling procedure. Initially, data on subject characteristics, lifestyle, socio-economic and health status were collected by means of a computer-aided personal interview. Subsequently, dietary intake was assessed within 14 days with three 24-hour diet recalls by telephone (two weekdays and one weekend day) using the software EPIC-SOFT $^{16}$. Within 6 weeks of recruitment all adults ( $\geq 18$ years) who completed a least one 24-hour dietary recall $(n=879)$ were invited to their nearest health office for blood sampling and standardised anthropometric measurements. Blood samples for further analysis of IgE and carotenoids, tocopherols and vitamin $\mathrm{C}$ were obtained from 568 participants. All participants gave their written informed consent. The study was approved by the local ethics committee.

\section{Allergic rbinitis and allergic sensitisation}

During the computer-aided personal interview, study participants were asked about 21 common diseases ('Have you ever had one of the following diseases diagnosed by a physician?'). Seventy-seven participants answered 'yes' to the question on allergic rhinitis. Because only 34 participants answered 'yes' to the question on asthma, we did not analyse this outcome variable in our study.

Allergic sensitisation to common aeroallergens (timothy, rye, birch, mugwort pollen, house dust mite Dermatophagoides pteronyssinus, cat and dog epithelia, Cladosporium herbarum mould) was assessed by specific serum IgE measurement using the CAPSX1 in vitro screening test (Pharmacia Upjohn, Uppsala, Sweden). Subjects with at least one specific $\operatorname{IgE} \geq 700 \mathrm{Ul}^{-1}$ were categorised as sensitised.

\section{Measurement of carotenoids, tocopherols, vitamin $C$ and cholesterol in plasma}

Aliquots of ethylenediaminetetraacetic acid-stabilised plasma samples were analysed for $\alpha$-carotene, $\beta$-carotene, lycopene, lutein/zeaxanthin, canthaxanthin and cryptoxanthin, as well as $\alpha$ - and $\gamma$-tocopherol, by means of highperformance liquid chromatography-ultraviolet/visible spectrophotometry according to a described method ${ }^{17}$. Calculations were made using the internal standard method and relative response factors. As internal standards, $\beta$-apo- 8 -carotenoic acid ethyl ester and DL- $\alpha$ tocopheryl acetate were added. Recovery of the standard substances from plasma samples was within 94 and 107\%, with coefficients of variation $<2.3 \%(n=5)$.

Vitamin C content of plasma samples was determined photometrically according to the method of Speitling et al. ${ }^{18}$. Ascorbic acid standard solution (Sigma, Deisenhofen, Germany) was taken for calibration and quality control. Mean recovery of ascorbic acid added to plasma samples $(n=7)$ was $101.6 \%$, with a coefficient of variation of $0.7 \%$.

Plasma total cholesterol concentration was analysed enzymatically using a standard test combination (Roche Diagnostics GmbH, Mannheim, Germany). For calibration and quality control Precinorm $\mathrm{L}^{\circledR}$ and C.f.a.s. Lipids ${ }^{\circledR}$, respectively, were used.

Due to analytical problems within one batch of samples, vitamin $\mathrm{C}$ concentrations of 17 samples are missing. In these samples, only half the amount of trichloroacetic acid necessary for protein precipitation was added. This mistake could not be corrected afterwards since precipitation should be done in fresh samples.

\section{Statistical analysis}

Continuous data are presented as mean \pm standard deviation (SD) and the Wilcoxon test was used to test for significant differences between women and men. Differences in categorical variables between women and men were assessed by the chi-square test. A two-tailed $P$-value of $<0.05$ was required for statistical significance.

The association between plasma carotenoid, tocopherol and vitamin $\mathrm{C}$ concentrations and allergic rhinitis or allergic sensitisation was assessed by using logistic regression models. For each micronutrient, plasma concentrations of the entire population were used to define cut-off points of quartiles. Odds ratios (OR) and 95\% confidence intervals (CI) were adjusted for sex, age, body mass index (BMI, $\mathrm{kg} \mathrm{m}^{-2}$ ), education (low/middle/high), smoking status (never/ex/current), sports activity in leisure time (yes/no) and season (spring/summer/autumn/winter). For the fat-soluble carotenoids and tocopherols we adjusted additionally for 
plasma cholesterol. Where statistically significant associations were found between allergic sensitisation and antioxidants in plasma we conducted an analysis stratified for age (participants 50 years or younger vs. older participants) and sex. Because there were only 20 men and 27 participants older than 50 years who suffered from allergic rhinitis in our study population, we analysed the association between allergic rhinitis and the antioxidants only in women and younger participants when results were significant. In an additional analysis we assessed the association between the ratios $\beta$-carotene/cholesterol and $\alpha$-tocopherol/cholesterol and allergic rhinitis and allergic sensitisation. $P$ for trend was assessed by setting the numerically coded quartiles as continuous variables in the model.

Correlations between dietary intake of $\beta$-carotene, $\alpha$-tocopherol and vitamin $\mathrm{E}$ (tocopherol equivalents) and concentrations of $\beta$-carotene and tocopherols in plasma were calculated using Spearman's correlation coefficients.

SAS version 8.2 was used for all calculations (SAS Institute, Cary, NC, USA).

\section{Results}

Table 1 shows the basic characteristics of the study population. Women were significantly younger and had lower BMI and energy intake than men. The prevalence of allergic rhinitis was nearly twice as high in women as in men, whereas the prevalence of allergic sensitisation did not differ significantly between sexes. Mean $( \pm S D)$ concentrations of the measured carotenoids, tocopherols and vitamin $\mathrm{C}$ in plasma are shown in Table 2. All differed significantly between sexes except for lutein/zeaxanthin. $\alpha$-Carotene, $\beta$-carotene, lutein/zeaxanthin, canthaxanthin and cryptoxanthin were right-skewed, whereas lycopene, $\alpha$-tocopherol, $\gamma$-tocopherol, vitamin $\mathrm{C}$ and the sum of carotenoids were normally distributed in both sexes.

Table 3 shows cut-offs for quartiles of the six measured carotenoids and their sum, $\alpha$ - and $\gamma$-tocopherol and vitamin $\mathrm{C}$ in plasma, and adjusted OR and corresponding 95\% CI for the association between these micronutrients and allergic rhinitis in the total population. We observed a negative association between the sum of carotenoids in plasma and allergic rhinitis $(P$ for trend $=0.0332$ ) which became stronger when we analysed only participants who were 50 years or younger, with OR $(95 \%$ CI) of 0.98 $(0.36-2.68)$ for the second, $0.67(0.24-1.87)$ for the third and $0.28(0.09-0.88)$ for the fourth quartile of plasma total carotenoids concentration ( $P$ for trend $=0.0218$, data not shown). Lycopene, the carotenoid which contributes the largest fraction to the sum of carotenoids, was also negatively associated with allergic rhinitis, although not reaching statistical significance ( $P$ for trend $=0.0608$ ).

Neither the other carotenoids nor the tocopherols or vitamin $\mathrm{C}$ showed any association with allergic rhinitis. Results did not change significantly when we calculated the ratios $\beta$-carotene/cholesterol and $\alpha$-tocopherol/cholesterol (data not shown).

The association between the six measured carotenoids and their sum, $\alpha$ - and $\gamma$-tocopherol and vitamin $C$ in plasma and allergic sensitisation in the total population is shown in Table 4. Consistent with our finding on allergic rhinitis, we observed a negative association between allergic sensitisation and the sum of carotenoids in plasma, but this was not statistically significant $(P$ for trend $=0.1492$ ). Risk for allergic sensitisation was significantly lowered in the fourth quartile of $\boldsymbol{\gamma}$-tocopherol concentration in plasma, compared with the other

Table 1 Characteristics of the study population

\begin{tabular}{|c|c|c|c|c|}
\hline & Total $(n=568)$ & Women $(n=325)$ & Men $(n=243)$ & $P$-value \\
\hline Age (years) & $48.5 \pm 15.3$ & $46.4 \pm 14.5$ & $51.3 \pm 15.7$ & 0.00019 \\
\hline Educational level $(\%)^{*}(n=548)$ & & & & $<0.0001 \|$ \\
\hline High & 21.7 & 18.5 & 26.1 & \\
\hline Medium & 31.2 & 38.8 & 20.9 & \\
\hline Low & 47.1 & 42.7 & 53.0 & \\
\hline Smoking status $(\%)(n=567)$ & & & & $<0.0001 \|$ \\
\hline Never smoker & 51.8 & 60.5 & 40.3 & \\
\hline Ex-smoker & 23.5 & 18.8 & 29.6 & \\
\hline Current smoker & 24.7 & 20.7 & 30.0 & \\
\hline Body mass index $\left(\mathrm{kg} \mathrm{m}^{-2}\right)$ & $27.1 \pm 5.0$ & $26.7 \pm 5.5$ & $27.6 \pm 4.2$ & 0.00029 \\
\hline Energy intake $\left(\mathrm{kcal}\right.$ day $\left.^{-1}\right)(n=561)$ & $1990 \pm 633$ & $1731 \pm 484$ & $2344 \pm 641$ & $<0.00019$ \\
\hline Cholesterol $\left(\mathrm{mmoll}^{-1}\right)$ & $5.39 \pm 0.98$ & $5.37 \pm 0.97$ & $5.42 \pm 0.99$ & 0.4182 च \\
\hline Asthma $(\%) \dagger$ & 6.0 & 7.7 & 3.7 & $0.0487 \|$ \\
\hline Hay fever (\%)‡ & 13.6 & 17.5 & 8.2 & $0.0013 \|$ \\
\hline Allergic sensitisation (\%)§ & 26.5 & 24.4 & 29.3 & $0.1863 \|$ \\
\hline
\end{tabular}

Data are expressed as mean \pm standard deviation or \%.

${ }^{*}$ High $->10$ classes; medium -10 classes; low $-<10$ classes.

†Defined as answering 'yes' to the question 'Has one of the following diseases ever been diagnosed by a doctor: ... asthma?' ‡Defined as answering 'yes' to the question 'Has one of the following diseases ever been diagnosed by a doctor: ... allergic rhinitis?'

§Defined as at least one specific immunoglobulin $\mathrm{E} \geq 700 \mathrm{UI}^{-1}$.

I Wilcoxon test.

\| Chi-square test. 
Table 2 Concentration $\left(\mu \mathrm{mol} \mathrm{I}^{-1}\right)$ of carotenoids, tocopherols and vitamin $\mathrm{C}$ in plasma samples of 568 adults

\begin{tabular}{lcccr}
\hline Nutrient & Total $(n=568)$ & Women $(n=325)$ & Men $(n=243)$ & $P$-value* \\
\hline Carotenoids & & & & \\
$\alpha$-Carotene & $0.095 \pm 0.101$ & $0.107 \pm 0.100$ & $0.079 \pm 0.101$ & $<0.0001$ \\
$\beta$-Carotene & $0.351 \pm 0.380$ & $0.394 \pm 0.365$ & $0.292 \pm 0.394$ & $<0.0001$ \\
Lycopene & $0.505 \pm 0.297$ & $0.529 \pm 0.306$ & $0.472 \pm 0.281$ & 0.0324 \\
Lutein/zeaxanthin & $0.247 \pm 0.131$ & $0.250 \pm 0.133$ & $0.243 \pm 0.127$ & 0.5028 \\
Canthaxanthin & $0.071 \pm 0.059$ & $0.065 \pm 0.055$ & $0.079 \pm 0.063$ & $<0.0001$ \\
Cryptoxanthin & $0.217 \pm 0.191$ & $0.253 \pm 0.222$ & $0.168 \pm 0.123$ & $<0.0001$ \\
Sum of carotenoids & $1.485 \pm 0.732$ & $1.599 \pm 0.754$ & $1.333 \pm 0.675$ & $<0.0001$ \\
Tocopherols & & & & \\
$\alpha$-Tocopherol & $30.380 \pm 9.980$ & $29.508 \pm 9.368$ & $31.547 \pm 10.653$ & 0.0100 \\
$\gamma$-Tocopherol & $1.222 \pm 0.663$ & $1.265 \pm 0.671$ & $1.165 \pm 0.649$ & 0.0329 \\
& $(n=551)$ & $(n=314)$ & $(n=237)$ & 0.0004 \\
Vitamin C & $78.031 \pm 22.124$ & $81.022 \pm 20.948$ & $74.069 \pm 23.046$ & \\
\hline
\end{tabular}

Data are expressed as mean \pm standard deviation.

${ }^{\star}$ Wilcoxon rank sum test for differences between men and women.

quartiles $(P$ for trend $=0.0410)$. After stratification for sex, this association was significant in men $(P$ for trend $=0.0186$ ) but not in women $(P$ for trend $=0.8437$, data not shown). After stratification for age, the negative association between $\gamma$-tocopherol and allergic sensitisation was significant in people aged 50 years or older $(P$ for trend $=0.0054$ ) but not in the younger age group ( $P$ for trend $=0.8829$ ). None of the single carotenoids,

Table 3 Odds ratio (OR) and corresponding 95\% confidence interval $(95 \% \mathrm{Cl})$ for the association between the concentration $\left(\mu \mathrm{moll}^{-1}\right)$ of carotenoids, tocopherols $(n=539)$ and vitamin C $(n=524)$ in plasma and allergic rhinitis*

\begin{tabular}{|c|c|c|c|c|c|}
\hline & 1st quartile & 2nd quartile† & 3rd quartile† & 4th quartile & $P$ for trend \\
\hline \multicolumn{6}{|l|}{ Carotenoids $\ddagger$} \\
\hline Cut-off for quartiles & $0-0.040$ & $0.040-0.070$ & $0.070-0.110$ & $0.110-1.060$ & \\
\hline Adj. OR $(95 \% \mathrm{Cl})$ & 1.00 & $2.93(1.34-6.43)$ & $1.75(0.74-4.13)$ & $1.44(0.60-3.44)$ & 0.8476 \\
\hline \multicolumn{6}{|l|}{$\beta$-Carotene } \\
\hline Cut-off for quartiles & $0-0.140$ & $0.140-0.250$ & $0.250-0.420$ & $0.402-3.540$ & \\
\hline Adj. OR $(95 \% \mathrm{Cl})$ & 1.00 & $1.03(0.47-2.26)$ & $1.10(0.49-2.47)$ & $0.81(0.35-1.87)$ & 0.6202 \\
\hline \multicolumn{6}{|l|}{ Lycopene } \\
\hline Cut-off for quartiles & $0-0.280$ & $0.280-0.452$ & $0.452-0.679$ & $0.679-2.070$ & \\
\hline Adj. OR (95\% Cl) & 1.00 & $0.68(0.33-1.43)$ & $0.56(0.26-1.21)$ & $0.47(0.21-1.05)$ & 0.0608 \\
\hline \multicolumn{6}{|l|}{ Lutein/zeaxanthin } \\
\hline Cut-off for quartiles & $0.030-0.160$ & $0.160-0.220$ & $0.220-0.300$ & $0.300-1.170$ & \\
\hline Adj. OR (95\% Cl) & 1.00 & $1.19(0.56-2.53)$ & $1.64(0.78-3.47)$ & $0.77(0.33-1.78)$ & 0.7812 \\
\hline \multicolumn{6}{|l|}{ Canthaxanthin } \\
\hline Cut-off for quartiles & $0-0.040$ & $0.040-0.060$ & $0.060-0.080$ & $0.080-0.550$ & \\
\hline Adj. OR $(95 \% \mathrm{Cl})$ & 1.00 & $1.43(0.76-2.70)$ & $0.57(0.23-1.44)$ & $0.89(0.39-2.05)$ & 0.4489 \\
\hline \multicolumn{6}{|l|}{ Cryptoxanthin } \\
\hline Cut-off for quartiles & $0.010-0.098$ & $0.098-0.160$ & $0.160-0.270$ & $0.270-1.940$ & \\
\hline Adj. OR $(95 \% \mathrm{Cl})$ & 1.00 & $1.52(0.72-3.24)$ & $0.62(0.26-1.49)$ & $0.86(0.37-2.01)$ & 0.2890 \\
\hline \multicolumn{6}{|l|}{ Sum of carotenoids } \\
\hline Cut-off for quartiles & $0.134-0.990$ & $0.990-1.348$ & $1.348-1.781$ & $1.781-5.720$ & \\
\hline Adj. OR $(95 \% \mathrm{Cl})$ & 1.00 & $1.13(0.54-2.39)$ & $0.72(0.33-1.58)$ & $0.44(0.19-1.03)$ & 0.0332 \\
\hline \multicolumn{6}{|l|}{$\begin{array}{l}\text { Tocopherols } \ddagger \\
\alpha \text {-Tocopherol }\end{array}$} \\
\hline Cut-off for quartiles & $10.05-24.48$ & $24.48-29.47$ & $29.47-34.43$ & $34.43-82.72$ & \\
\hline Adj. OR (95\% Cl) & 100 & $1.07(0.52-2.22)$ & $0.98(0.43-2.20)$ & $1.24(0.51-3.02)$ & 0.7071 \\
\hline \multicolumn{6}{|l|}{$\gamma$-Tocopherol } \\
\hline Cut-off for quartiles & $0.210-0.752$ & $0.752-1.100$ & $1.100-1.558$ & $1.558-4.860$ & \\
\hline Adj. OR (95\% Cl) & 1.00 & $0.79(0.36-1.74)$ & $1.03(0.49-2.17)$ & $0.77(0.35-1.71)$ & 0.6831 \\
\hline \multicolumn{6}{|l|}{ Vitamin $C \S$} \\
\hline Cut-off for quartiles & $11.81-63.78$ & $63.78-78.50$ & $78.50-91.04$ & $91.04-157.18$ & \\
\hline Adj. OR $(95 \% \mathrm{Cl})$ & 1.00 & $1.43(0.66-3.10)$ & $0.95(0.41-2.21)$ & $1.22(0.54-2.76)$ & 0.9383 \\
\hline
\end{tabular}

*Defined as answering 'yes' to the question 'Has a doctor ever diagnosed one of the following diseases: ... allergic rhinitis?' †First quartile was set as the reference category.

$\ddagger$ Adjusted for sex, age (continuous), education (low, middle, high), smoking status (never, ex, current), body mass index (continuous), sports activity (yes/no), season (spring, summer, autumn, winter) and plasma cholesterol $\left(\mathrm{mmoll}^{-1}\right)$.

$\S$ Adjusted for sex, age (continuous), education (low, middle, high), smoking status (never, ex, current), body mass index (continuous), sports activity (yes/no) and season (spring, summer, autumn, winter). 
Table 4 Odds ratio (OR) and corresponding 95\% confidence interval $(95 \% \mathrm{Cl})$ for the association between the concentration $\left(\mu \mathrm{molI}^{-1}\right)$ of carotenoids, tocopherols $(n=537)$ and vitamin $\mathrm{C}(n=523)$ in plasma and allergic sensitisation*

\begin{tabular}{|c|c|c|c|c|c|}
\hline & 1st quartile & 2nd quartile† & 3rd quartile† & 4th quartile $\dagger$ & $P$ for trend \\
\hline \multicolumn{6}{|l|}{$\begin{array}{l}\text { Carotenoids } \neq \\
\alpha \text {-Carotene }\end{array}$} \\
\hline Cut-off for quartiles & $0-0.040$ & $0.040-0.070$ & $0.070-0.110$ & $0.110-1.060$ & \\
\hline Adj. OR $(95 \% \mathrm{Cl})$ & 1.00 & $1.43(0.80-2.58)$ & $1.56(0.85-2.88)$ & $1.27(0.68-2.40)$ & 0.4151 \\
\hline \multicolumn{6}{|l|}{$\beta$-Carotene } \\
\hline Cut-off for quartiles & $0-0.140$ & $0.140-0.250$ & $0.250-0.420$ & $0.402-3.540$ & \\
\hline Adj. OR (95\% Cl) & 1.00 & $0.80(0.44-1.47)$ & $0.89(0.47-1.67)$ & $0.82(0.43-1.56)$ & 0.6600 \\
\hline \multicolumn{6}{|l|}{ Lycopene } \\
\hline Cut-off for quartiles & $0-0.280$ & $0.280-0.452$ & $0.452-0.679$ & $0.679-2.070$ & \\
\hline Adj. OR (95\% Cl) & 1.00 & $0.79(0.43-1.47)$ & $0.78(0.42-1.45)$ & $0.79(0.42-1.48)$ & 0.5046 \\
\hline \multicolumn{6}{|l|}{ Lutein/zeaxanthin } \\
\hline Cut-off for quartiles & $0.030-0.160$ & $0.160-0.220$ & $0.220-0.300$ & $0.300-1.170$ & \\
\hline Adj. OR $(95 \% \mathrm{Cl})$ & 1.00 & $1.27(0.70-2.30)$ & $1.22(0.66-2.26)$ & $0.78(0.40-1.50)$ & 0.4370 \\
\hline \multicolumn{6}{|l|}{ Canthaxanthin } \\
\hline Cut-off for quartiles & $0-0.040$ & $0.040-0.060$ & $0.060-0.080$ & $0.080-0.550$ & \\
\hline Adj. OR $(95 \% \mathrm{Cl})$ & 1.00 & $0.86(0.50-1.49)$ & $0.81(0.43-1.53)$ & $0.75(0.40-1.37)$ & 0.3366 \\
\hline \multicolumn{6}{|l|}{ Cryptoxanthin } \\
\hline Cut-off for quartiles & $0.010-0.098$ & $0.098-0.160$ & $0.160-0.270$ & $0.270-1.940$ & \\
\hline Adj. OR $(95 \% \mathrm{Cl})$ & 1.00 & $1.43(0.78-2.62)$ & $0.89(0.46-1.73)$ & $0.88(0.45-1.72)$ & 0.3412 \\
\hline \multicolumn{6}{|l|}{ Sum of carotenoids } \\
\hline Cut-off for quartiles & $0.134-0.990$ & $0.990-1.348$ & $1.348-1.781$ & $1.781-5.720$ & \\
\hline Adj. OR $(95 \% \mathrm{Cl})$ & 1.00 & $0.66(0.35-1.23)$ & $0.92(0.50-1.69)$ & $0.53(0.27-1.03)$ & 0.1492 \\
\hline \multicolumn{6}{|l|}{$\begin{array}{l}\text { Tocopherolsł } \\
\alpha \text {-Tocopherol }\end{array}$} \\
\hline Cut-off for quartiles & $10.05-24.48$ & $24.48-29.47$ & $29.47-34.43$ & $34.43-82.72$ & \\
\hline Adj. OR $(95 \% \mathrm{Cl})$ & 1.00 & $1.08(0.61-1.93)$ & $0.73(0.39-1.39)$ & $0.67(0.33-1.36)$ & 0.1795 \\
\hline \multicolumn{6}{|l|}{$\gamma$-Tocopherol } \\
\hline Cut-off for quartiles & $0.210-0.752$ & $0.752-1.100$ & $1.100-1.558$ & $1.558-4.860$ & \\
\hline Adj. OR (95\% Cl) & 1.00 & $0.92(0.51-1.65)$ & $1.00(0.56-1.80)$ & $0.45(0.23-0.88)$ & 0.0410 \\
\hline \multicolumn{6}{|l|}{ Vitamin $C \S$} \\
\hline Cut-off for quartiles & $11.81-63.78$ & $63.78-78.50$ & $78.50-91.04$ & $91.04-157.18$ & \\
\hline Adj. OR $(95 \% \mathrm{Cl})$ & 1.00 & $1.18(0.64-2.17)$ & $1.02(0.54-1.91)$ & $1.44(0.77-2.67)$ & 0.3353 \\
\hline
\end{tabular}

* Defined as at least one specific immunoglobulin $\mathrm{E} \geq 700 \mathrm{UI}^{-1}$

$\dagger$ First quartile was set as the reference category.

$\ddagger$ Adjusted for sex, age (continuous), education (low, middle, high), smoking status (never, ex, current), body mass index (continuous), sports activity (yes/no), season (spring, summer, autumn, winter) and plasma cholesterol (mmol $\mathrm{I}^{-1}$ ).

$\S$ Adjusted for sex, age (continuous), education (low, middle, high), smoking status (never, ex, current), body mass index (continuous), sports activity (yes/no) and season (spring, summer, autumn, winter).

$\alpha$-tocopherol or vitamin C in plasma showed a significant influence on allergic sensitisation. Results did not change significantly when we restricted the analysis to people younger than 50 years (data not shown).

Calculating the ratios $\beta$-carotene/cholesterol and $\alpha$-tocopherol/cholesterol did not significantly alter the results for these two micronutrients (data not shown).

Dietary intake of $\beta$-carotene, $\alpha$-tocopherol or vitamin $\mathrm{E}$ (tocopherol equivalents) was not associated with allergic rhinitis or allergic sensitisation (data not shown).

The correlation between dietary intake and plasma concentration of the analysed nutrients was weak, with crude correlation coefficients of 0.14 for $\beta$-carotene and -0.01 for $\alpha$-tocopherol. The crude correlation coefficient for vitamin $\mathrm{E}$ (tocopherol equivalents) intake and plasma $\alpha$-tocopherol was 0.02 , for vitamin $\mathrm{E}$ intake and plasma $\gamma$-tocopherol was 0.01 , and for vitamin $\mathrm{E}$ intake and the sum of $\alpha$-tocopherol $+\gamma$-tocopherol in plasma was 0.02.

Relating plasma concentrations of $\beta$-carotene and the tocopherols to plasma cholesterol, correlation coefficients were 0.13 for $\beta$-carotene, 0.10 for $\alpha$-tocopherol, 0.12 for vitamin E (tocopherol equivalents) intake and plasma $\alpha$-tocopherol, 0.05 for vitamin $\mathrm{E}$ intake and plasma $\gamma$-tocopherol, and 0.13 for vitamin $E$ intake and the sum of plasma $\alpha$-tocopherol $+\gamma$-tocopherol. Additionally, we analysed triglycerides in plasma from a sub-sample of 200 participants. Relating plasma concentrations of the antioxidants to plasma triglycerides, correlation coefficients were 0.11 for $\beta$-carotene, 0.04 for $\alpha$-tocopherol, 0.05 for vitamin $\mathrm{E}$ (tocopherol equivalents) intake and plasma $\alpha$-tocopherol, 0.05 for vitamin $\mathrm{E}$ intake and plasma $\gamma$-tocopherol, and 0.05 for vitamin E intake and the sum of plasma $\alpha$-tocopherol $+\gamma$-tocopherol (data not shown).

\section{Discussion}

In this study we investigated the association between the prevalence of allergic diseases in an adult population and plasma concentrations of carotenoids, tocopherols and vitamin C. Due to uncertainties in the bioavailability of carotenoids, concentrations of these compounds in plasma gives more valid information on the biologically 
available amount. Concentrations of $\gamma$-tocopherol in plasma were slightly lower, and concentrations of $\alpha$-tocopherol and vitamin $\mathrm{C}$ were slightly higher, than recently reported from a combined analysis of data from five European centres ${ }^{19}$. Compared with other studies, the plasma carotenoid concentrations in our study population are comparable to the lowest in Europe ${ }^{19,20}$. To our knowledge this study is the first that analyses the association between plasma concentrations of these micronutrients and allergic rhinitis in adults. The sum of measured carotenoids was significantly negatively associated with allergic rhinitis and not significantly associated with allergic sensitisation. Although not reaching statistical significance, lycopene was also negatively associated with allergic rhinitis, which contributes to the overall carotenoid result. Between $\alpha$-carotene in plasma and allergic rhinitis we found a positive association in the second quartile with an OR (95\% CI) of 2.93 (1.34-6.43), whereas the odds were decreasing in the third and fourth quartiles, $P$ for trend being 0.8476 . This observation might be due to measurement error, because plasma concentrations of $\alpha$-carotene were relatively low in our study population. Whereas $\beta$-carotene showed no influence on allergic rhinitis in our study, Nagel et al. found a positive association between $\beta$-carotene intake over the last year before recruitment and adult-onset hay fever in ex- and current smokers, but not in non-smokers ${ }^{15}$. Because the number of smokers with allergic rhinitis was too small in our study, we could not stratify for smokers and nonsmokers. Allergic sensitisation, assessed by skin prick test, was negatively associated with serum $\alpha$-carotene and cryptoxanthin and positively associated with serum lycopene in a large US study population ${ }^{13}$ but not in our study. Carotenoid levels in blood are sensitive markers for dietary intake, because they are not closely regulated by homeostatic mechanisms ${ }^{21}$. However, several factors influence carotenoid concentrations in plasma; for example, the mode of preparation of food and the composition of the diet have an important impact on the bioavailability of carotenoids. Smoking, high ethanol consumption and gender modify their metabolism ${ }^{22}$. Therefore, correlations between dietary intake and blood levels were only modest $(r<0.5)$ in most studies ${ }^{10}$. A high concentration of the sum of six carotenoids in plasma, which was negatively associated with allergic rhinitis in our study, might be an indicator for a diet rich in various fruits and vegetables. Possibly, additive and synergistic effects of bioactive compounds in fruit and vegetables might be responsible for our findings. Negative associations between fruit consumption and allergic diseases have been reported from several studies ${ }^{23-26}$.

Whereas plasma $\alpha$-tocopherol was not associated with allergic rhinitis or sensitisation, we found a negative association between $\boldsymbol{\gamma}$-tocopherol and allergic sensitisation. In a study on a US population, serum vitamin E was negatively associated with allergic sensitisation assessed by skin prick test ${ }^{13}$, which is consistent with the results of the study of Fogarty et al., who observed a negative association between dietary intake of vitamin $\mathrm{E}$ and allergic sensitisation $^{14}$. Nagel et al. observed a decreased risk of adult-onset hay fever with increasing intake of vitamin $\mathrm{E}^{15}$. On the other hand, Woods et al. found no association between dietary intake of vitamin $\mathrm{E}$ and atopy assessed by skin prick test ${ }^{24}$. In a clinical study patients with seasonal allergic rhinitis reported an improvement in the nasal symptoms after high doses of vitamin $\mathrm{E}$ $\left(800 \mathrm{mg} \mathrm{day}^{-1}\right)$, but this was not confirmed by the investigators who evaluated the patients. The percentage of days with serious symptoms or use of medications was not reduced after vitamin $\mathrm{E}$ supplementation ${ }^{27}$. One possible explanation for the inconsistent results on $\alpha$-tocopherol and vitamin $\mathrm{E}$ respectively is the selective incorporation of $\alpha$-tocopherol into very-low-density lipoproteins. A specific protein in the liver, $\alpha$-tocopherol transfer protein, selectively sorts out $\alpha$-tocopherol from other tocopherols ${ }^{28}$ and, therefore, the concentration of $\alpha$-tocopherol in plasma reflects dietary intake of $\alpha$-tocopherol to a very modest degree ${ }^{10,21,28}$. The protective effects of high intake of vitamin $\mathrm{E}$ on allergic diseases observed in epidemiological studies might be a marker for a certain lifestyle including high consumption of fruits and vegetables, vegetable oils and whole-grain products. Another explanation might be that $\alpha$ - and $\gamma$-tocopherol have different effects on allergic diseases. In studies conducted so far, associations have only been assessed between vitamin $\mathrm{E}$ and $\alpha$-tocopherol, respectively, and allergic diseases, but not between $\boldsymbol{\gamma}$-tocopherol and these diseases. In the fourth quartile of plasma $\gamma$-tocopherol concentration we observed a significantly decreased risk for allergic sensitisation. $\gamma$-Tocopherol is thought to be more effective in detoxifying nitrogen dioxide and exhibits greater anti-inflammatory effects than $\alpha$-tocopherol in vitro ${ }^{29-32}$. In Northern America, where $\gamma$-tocopherol accounts for approximately $70 \%$ of the vitamin $\mathrm{E}$ in the $\operatorname{diet}^{31,32}$, serum concentrations are much higher than in our population. Huang et al. reported a median serum $\gamma$-tocopherol concentration of $2.30 \mu \mathrm{moll}^{-1}$ in their study population ${ }^{32}$, a concentration which is achieved by only $7 \%$ of our study population (median concentration of $1.10 \mu \mathrm{moll}^{-1}$ ). A recently published nested case-control study on breast cancer with women enrolled in the Nurses' Health Study measured a median plasma $\gamma$-tocopherol concentration of $4.39 \mathrm{mmoll}^{-1}$ in the control group ${ }^{33}$, which is achieved by only one woman in our study. Median concentrations of $\alpha$-tocopherol were slightly lower in the two US studies at 27.2 and $26.69 \mu \mathrm{moll}^{-1}$ (reference 32 and 33, respectively). Therefore, concentrations of $\gamma$-tocopherol in our study population might be too low to see effects already in the second and third quartiles and further effects in all subgroups by gender or age. McKeever et al. ${ }^{13}$ conducted their study on a US population with a probably 
higher dietary intake and proportion of $\gamma$-tocopherol in serum than our study population, which might be the explanation for the negative association they observed between serum vitamin $\mathrm{E}$ and allergic sensitisation.

In our study plasma vitamin $\mathrm{C}$ showed no association with allergic rhinitis or allergic sensitisation, which is consistent with the results of McKeever et al. on the association between serum vitamin $\mathrm{C}$ and allergic sensitisation $^{13}$. Studies on dietary intake of vitamin C also failed to find an association with adult-onset hay fever $^{15}$ and allergic sensitisation ${ }^{23}$.

There are some limitations of our study that should be considered. Because of the cross-sectional nature of our study we cannot exclude reverse causality. We measured vitamins in plasma at one point in time, which does not necessarily reflect vitamin status at the time the disease evolved. Participants might have changed their diet after the disease occurred or vitamin status might be affected by the disease itself. Participants were not necessarily fasting when blood samples were drawn. The age of our study population ranges from 18 to 81 years, which is very large, but we do not think that this causes a major problem. In an age-stratified analysis on allergic sensitisation results did not differ significantly between people who were 50 years or older and younger people, except for $\gamma$-tocopherol. A critical point is the low prevalence of allergic rhinitis in men, which is only half that of women, whereas allergic sensitisation is more prevalent in men, but we have no explanation for this difference. We conducted a secondary analysis that was not planned when the study was designed. Therefore, exact calculations on sample size and power have not been conducted in this analysis and our results need to be interpreted with caution.

Summarising the results, our finding of a negative association between the sum of six measured carotenoids and allergic rhinitis suggests that a high intake of various fruit and vegetables might reduce the risk for allergic diseases. The results of a negative association between $\gamma$-tocopherol, but not $\alpha$-tocopherol, in plasma might signify a different role of these two tocopherols in allergic sensitisation. It would be interesting to investigate the two tocopherols separately in future studies, especially in countries with high dietary intake of $\boldsymbol{\gamma}$-tocopherol.

\section{Acknowledgements}

We very much appreciate the co-operation of the study participants as well as the work of all co-workers involved in the sampling of data and blood samples. We especially thank the physicians from the health offices in Bavaria for providing study rooms and for drawing the blood samples, and Drs Weidner and Gabrio, Landesgesundheitsamt Baden-Wuerttemberg, for IgE measurements.

This work was funded by the Kurt-Eberhard-BodeStiftung and the Bavarian Ministry of Environment, Health and Consumer Protection.

\section{References}

1 Burr ML, Butland BK, King S, Vaughan-Williams E. Changes in asthma prevalence: two surveys 15 years apart. Archives of Disease in Childhood 1989; 64: 1452-6.

2 Anderson H, Butland B, Strachan D. Trends in prevalence and severity of childhood asthma. British Medical Journal 1994; 308: 1600-4.

3 Braun-Fahrlander C, Gassner M, Grize L, Takken-Sahli K, Neu U, Stricker T, et al. No further increase in asthma, hay fever and atopic sensitisation in adolescents living in Switzerland. European Respiratory Journal 2004; 23: $407-13$.

4 Ronchetti R, Villa MP, Barreto M, Rota R, Pagani J, Martella S, et al. Is the increase in childhood asthma coming to an end? Findings from three surveys of schoolchildren in Rome, Italy. European Respiratory Journal 2001; 17: 881-6.

5 Verlato G, Corsico A, Villani S, Cerveri I, Migliore E, Accordini S, et al. Is the prevalence of adult asthma and allergic rhinitis still increasing? Results of an Italian study. Journal of Allergy and Clinical Immunology 2003; 111: $1232-8$.

6 Fogarty A, Britton J. The role of diet in the aetiology of asthma. Clinical and Experimental Allergy 2000; 30: $615-27$.

7 Soutar A, Seaton A, Brown K. Bronchial reactivity and dietary antioxidants. Thorax 1998; 52: 166-70.

8 Monteleone CA, Sherman AR. Nutrition and asthma. Archives of Internal Medicine 1997; 157: 23-34.

9 Bowler RP, Crapo JD. Oxidative stress in allergic respiratory diseases. Journal of Allergy and Clinical Immunology 2002; 110: $349-56$.

10 Mayne ST. Antioxidant nutrients and chronic disease: use of biomarkers of exposure and oxidative stress status in epidemiologic research. Journal of Nutrition 2003; 133: 933S-40S

11 McKeever TM, Britton J. Diet and asthma. American Journal of Respiratory and Critical Care Medicine 2004; 170: 725-9.

12 Li-Weber M, Giaisi M, Treiber MK, Krammer PH. Vitamin E inhibits IL- 4 gene expression in peripheral blood T cells. European Journal of Immunology 2002; 32: 2401-8.

13 McKeever TM, Lewis SA, Smit H, Burney P, Britton J, Cassano PA. Serum nutrient markers and skin prick testing using data from the Third National Health and Nutrition Examination Survey. Journal of Allergy and Clinical Immunology 2004; 114: 1398-402.

14 Fogarty A, Lewis S, Weiss S, Britton J. Dietary vitamin E, IgE concentrations, and atopy. Lancet 2000; 356: 1573-4.

15 Nagel G, Nieters A, Becker N, Linseisen J. The influence of the dietary intake of fatty acids and antioxidants on hay fever in adults. Allergy 2003; 58: 1277-84.

16 Slimani N, Ferrari P, Ocké M, Welch A, Boeing H, Liere M, et al. Standardization of the 24-hour diet recall calibration method used in the European Prospective Investigation into Cancer and Nutrition (EPIC): general concepts and preliminary results. European Journal of Clinical Nutrition 2000; 54: 900-17.

17 Hess D, Keller HE, Oberlin B, Bonfanti F, Schüep W. Simultaneous determination of retinol, tocopherols, carotenes and lycopene in plasma by means of high-performance liquid chromatography on reversed phase. International Journal for Vitamin and Nutrition Research 1991; 61: 232-8.

18 Speitling A, Hüppe R, Kohlmeier M, Matiaske B, Stelte W, Thefeld W, et al. Methodenhandbuch VERA. In: Kübler W, Anders HJ, Heeschen W, eds. VERA Series Vol. I. Niederkleen: Wissenschaftlicher Fachverlag Dr Fleck, 1992; $100-2$.

19 Olmedilla B, Granado F, Southon S, Wright AJA, Blanco I, Gil-Martinez E, et al. Serum concentrations of carotenoids 
and vitamins A, E, and C in control subjects from five European countries. British Journal of Nutrition 2001; 85: 227-38.

20 Al-Delaimy WK, van Kappel AL, Ferrari P, Slimani N, Steghens JP, Bingham S, et al. Plasma levels of six carotenoids in nine European countries: report from the European Prospective Investigation into Cancer and Nutrition (EPIC). Public Health Nutrition 2004; 7: 713-22.

21 Hunter D. Biochemical indicators of dietary intake. In: Willett W, ed. Nutritional Epidemiology. Oxford: Oxford University Press, 1998; 174-243.

22 Parker RS, Swanson JE, You CS, Edwards AJ, Huang T. Bioavailability of carotenoids in human subjects. Proceedings of the Nutrition Society 1999; 58: 155-62.

23 Forastiere F, Pistelli R, Sestini P, Fortes C, Renzoni E, Rusconi F, et al. Consumption of fresh fruit rich in vitamin $\mathrm{C}$ and wheezing symptoms in children. SIDRIA Collaborative Group, Italy (Italian Studies on Respiratory Disorders in Children and the Environment). Thorax 2000; 55: 283-8.

24 Woods RK, Walters EH, Raven JM, Wolfe R, Ireland PD, Thien FC, et al. Food and nutrient intakes and asthma risk in young adults. American Journal of Clinical Nutrition 2003; 78: $414-21$.

25 Heinrich J, Hoelscher B, Bolte G, Winkler G. Allergic sensitization and diet: ecological analysis in selected European cities. European Respiratory Journal 2001; 17: 395-402.

26 Ellwood P, Asher MI, Bjoerksten B, Burr M, Pearce N, Robertson CF and the ISAAC Phase One Study Group. Diet and asthma, allergic rhinoconjunctivitis and atopic eczema symptom prevalence: an ecological analysis of the International Study of Asthma and Allergies in Childhood
(ISAAC) data. European Respiratory Journal 2000; 17: 436-43.

27 Shahar E, Hassoun G, Pollack S. Effect of vitamin E supplementation on the regular treatment of seasonal allergic rhinitis. Annals of Allergy, Asthma \& Immunology 2004; 92: 654-8.

28 Brigelius-Flohé R, Kelly FJ, Salonen JT, Neuzil J, Zingg JM, Azzi A. The European perspective on vitamin E: current knowledge and future research. American Journal of Clinical Nutrition 2002; 76: 703-16.

29 Christen S, Woodall AA, Shigenaga MK, Southwell-Keely PT, Duncan MW, Ames BN. $\gamma$-Tocopherol traps mutagenic electrophiles such as $\mathrm{NO}_{\mathrm{x}}$ and complements $\alpha$-tocopherol: physiological implications. Proceedings of the National Academy of Sciences of the USA 1997; 94: 3217-22.

30 Jiang Q, Christen S, Shigenaga MK, Ames BN. $\boldsymbol{\gamma}$-Tocopherol, the major form of vitamin $\mathrm{E}$ in the US diet, deserves more attention. American Journal of Clinical Nutrition 2001; 74 : $714-22$.

31 Jiang Q, Elson-Schwab I, Courtemanche C, Ames BN. $\gamma$-Tocopherol and its major metabolite, in contrast to $\alpha$-tocopherol, inhibit cyclooxygenase activity in macrophages and epithelial cells. Proceedings of the National Academy of Sciences of the USA 2000; 97: 11494-9.

32 Huang HY, Appel LJ. Supplementation of diets with $\alpha$-tocopherol reduces serum concentrations of $\gamma$ - and $\delta$-tocopherol in humans. Journal of Nutrition 2003; 133: $3137-40$.

33 Tamimi RM, Hankinson SE, Campos H, Spiegelman D, Zhang S, Colditz GA, et al. Plasma carotenoids, retinol, and tocopherols and risk of breast cancer. American Journal of Epidemiology 2005; 161: 153-60. 